\title{
Development of a nerve stretcher for in vivo stretching of nerve fibres
}

\author{
Muhammad Sana Ullah Sahar ${ }^{1}$, Tamer Mettyas ${ }^{2}, \&$ \\ Matthew Barton ${ }^{3,4,5}$ \\ ${ }^{1}$ School of Engineering and Built Environment, Griffith University, Australia. \\ ${ }^{2}$ Royal Brisbane and Women's Hospital, Queensland, Australia. \\ ${ }^{3}$ Clem Jones Centre for Neurobiology and Stem Cell Therapies, Griffith \\ University, Australia. \\ ${ }^{4}$ Menzies Health Institute Queensland, Griffith University, Australia. \\ 5School of Nursing and Midwifery, Griffith University, Australia. \\ E-mail: m.sahar@griffithuni.edu.au
}

June 2018

\begin{abstract}
Axons in vitro respond to mechanical stimulus and can be stretched mechanically to increase their rate of growth. This type of accelerated growth under the influence of tensile forces alone appears independent of chemical cues and growth cones. The stretch-growth of axonal tracts ex vivo and their transient lengthening have been discussed in literature extensively; however; evidence of in vivo investigations is scarce. Stretching axons, although practical ex vivo, is more challenging in vivo due to the difficulties of applying in situ axial tensile forces. Here, a technique has been developed to apply axial tensile forces to a peripheral nerve in vivo. A device has been constructed, called a Nerve Stretcher, which makes use of negative gauge pressure to pull sectioned nerve stumps in a confined nerve prosthesis. This article presents the development of this device and a discussion of the methodology used to hold sciatic nerve stumps in a Tshaped nerve prosthesis. The findings of this study will form the basis of future nerve-stretch growth studies.
\end{abstract}

Keywords: Peripheral nerve, axonal stress, nerve stretching, stretch-growth, axon stretching. 


\section{Introduction}

Neuronal response to mechanical stimuli - a phenomenon known as mechanotransduction - plays a vital role in its growth and development. Once neurons reach their respective target, further growth depends on mechanical forces that generally arise as the organism grows in size $[1,2]$. Although the idea of axonal stretch-growth is not new, it is nevertheless relatively understudied. Soon after Paul Weiss coined the term towed-growth and described the possibility of axonal stretch-growth [3] in vitro, the response of axons to mechanical stretch has been reported continually in the literature. Since then, many ex vivo pioneer studies at tissue level demonstrated that axons produced impressive growth under the effect of purely mechanical tensile forces. For instance, dorsal root ganglion (DRG) neurons from a chick embryo were stretched at a rate of between 0.04 and $0.17 \mathrm{~mm} /$ hour to achieve a $1 \mathrm{~mm}$ axonal extension without displaying a bottleneck effect after 24 hours of stretch [4]. A linear relationship was found between tension and growth rate when chicken sensory neurites were subjected to axial tensile force $[5,6]$; however, these axons thinned when stretched and thickened when stationary [7]. Neurites of pheochromocytoma (PC-12) cells also showed a linear relationship between axial tension and induced strain with a high degree of reproducibility [8]. Similarly, stretching primary rat cortex neurons at $0.042 \mathrm{~mm} /$ hour over 10 days, produced a $10 \mathrm{~mm}$ increase in their length [9]. Among neural tissues, rat DRGs showed the greatest stretchgrowth rate of $1 \mathrm{~mm} /$ day; however, stretch rates beyond $1 \mathrm{~mm} /$ day resulted in axon disconnection $[10,11]$. Interestingly, as a result of stretch-growth, neurons not only increased in length but also retained their ability to transmit action potentials [12].

Axonal stretch-growth studies making use of force calibrated glass needles to stretch axons $[5,8]$ were employed ex vivo, but to the best of our knowledge, studies investigating in vivo nerve stretch-growth is absent. The underlying reason, aforementioned, is that in vivo axonal stretch-growth is challenging because of the difficulty of stretching a nerve fibre, in situ, without inflicting trauma. Notwithstanding, here a method of applying axial tensile forces to a peripheral nerve, in vivo has been presented. A device which we have named nerve stretcher, was constructed to pull rat sciatic nerve sections, within a custom-manufactured T-shaped silicone nerve prosthesis by using a controlled force.

\section{Scope of the manuscript}

The present study is a proof of concept to translate the idea of stretching individual axons in vitro $[1,4,10]$ to stretching an entire nerve in vivo. So, this manuscript briefly explains (a) the development of such a device that can provide such translation, and (b) presents a pilot study with an in vivo experimental model to investigate a feasible way of applying negative pressure to stretch whole sectioned peripheral nerves and testing the stability of developed platform. The results of this pilot study will be used to inform our subsequent research to investigate the outcome of negative pressure on axon and nerve regeneration.

\section{Methods and Materials}

\subsection{Device concept and working principle}

The nerve stretcher consists of a micro vacuum pump (Skoocom Electronic Co., Ltd, CN) operating at 3 volts and capable of producing a maximum of $\approx 200$ $\mathrm{mmHg}\left(0.027 \mathrm{~N} / \mathrm{mm}^{2}\right)$ vacuum (gauge) in an acrylic vacuum chamber (V.A.C. Freedom $300 \mathrm{ml}$ Canister, KCI Medical Ltd, IRL). The pump is connected to the vacuum chamber via silicone tubing (Part No. T2011, Qosina Co., USA). The vacuum chamber incorporates a sachet of silica gel to absorb any moisture/fluid entering the chamber. The level of vacuum in the vacuum chamber is monitored by a vacuum sensor (NXP Semiconductors, Inc. NLD) and is maintained at a specified level by a microcontroller (Teensy 3.6, Teensy Inc. USA). The vacuum sensor is an analogue sensor, and it produces a continuous output signal (voltage) proportional to the level of vacuum. The microcontroller required the use of its dedicated 16 bits analogue-to-digital converter to convert incoming analogue signal to a digital signal. The microcontroller also provides a useful platform to control various devices such as a vacuum pump, solenoid valve, quadrature encoder, and a graphics display. The working principle of the nerve stretcher is displayed in figure 1.

The device is programmed through a custom control module written in the C-language (see figure 2 for pseudo-code). The desired level of vacuum $\left(\mathrm{P}_{\text {set }}\right)$ can be set by rotating the knob of the rotary quadrature encoder. Upon turning the device ON, the microcontroller initialises by sending a signal to the solenoid valve which lets filtered air enter the vacuum 


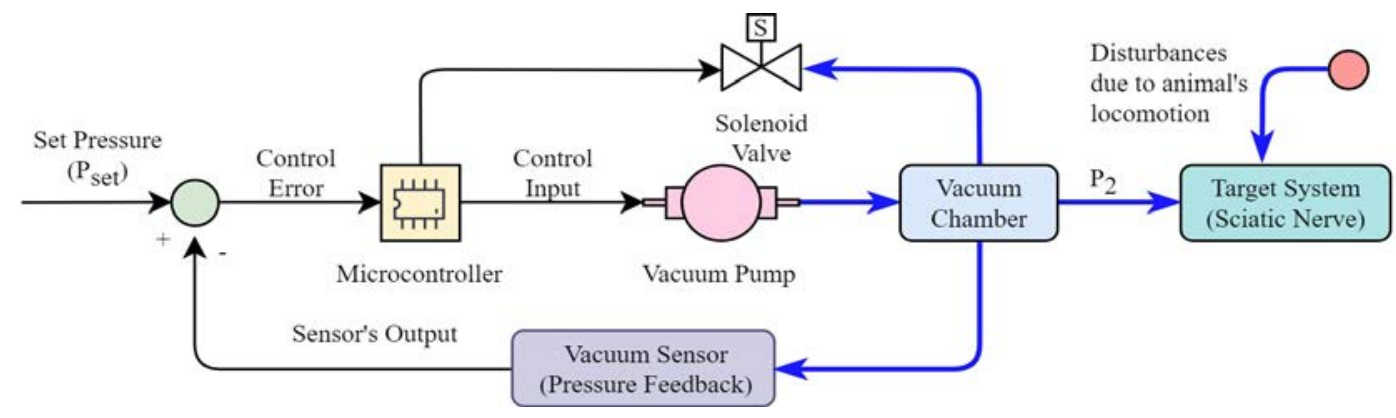

Figure 1. The basic working principle of the nerve stretcher (Black lines represents electrical signals and blue lines represents negative pressure flow).

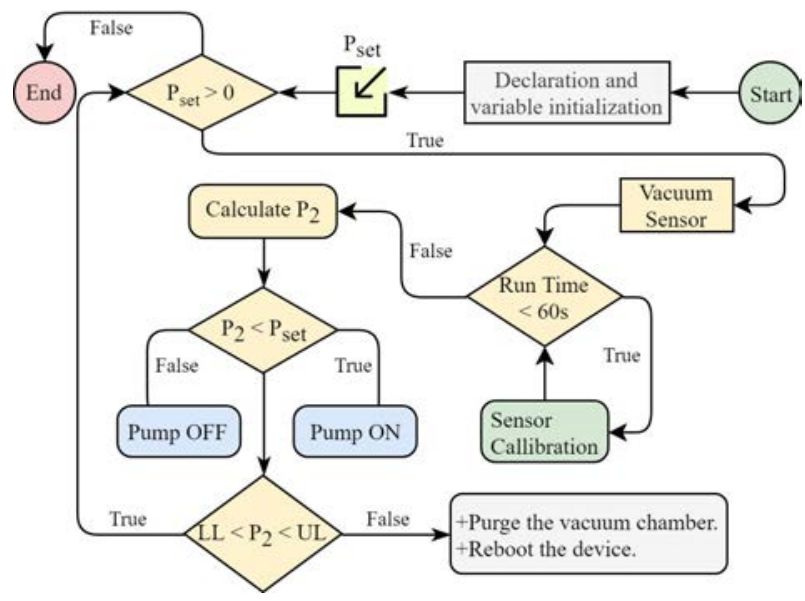

Figure 2. The flow chart of the nerve stretcher $\left(\mathrm{P}_{\text {set }}=\right.$ Desired pressure, $\mathrm{P}_{2}=$ The amount of negative pressure developed in the vacuum chamber, $\mathrm{LL}=$ Lower limit, $\mathrm{UL}=$ Upper limit).

chamber allowing the pressure inside the vacuum chamber to reach atmospheric pressure. This one-time step at device startup is crucial as the initial pressure level inside the vacuum chamber provides a reference point for automatic calibration of the vacuum sensor. The solenoid valve also acts as a vacuum breaker if the level of vacuum elevates erroneously due to sensor drift. The microcontroller then repeatedly performs the following tasks; (i) polling the sensor to measure the level of vacuum in the vacuum chamber, (ii) comparing the current level vacuum $\left(\mathrm{P}_{2}\right)$ against the vacuum set point $\left(\mathrm{P}_{\text {set }}\right)$ and (iii) finally turning the vacuum pump on or off as required to maintain a steady state of vacuum in the vacuum chamber. The vacuum $\left(\mathrm{P}_{2}\right)$ generated in the vacuum chamber can then be utilised for nerve stretching. The vacuum $\left(\mathrm{P}_{2}\right)$ was recorded every minute on a memory card (Samsung Electronics Co., Ltd. KR) attached to the controller to keep track of the device performance.

\subsection{Fabrication of a T-shaped nerve prosthesis}

Subject to the available space at the implantation site (sciatic nerve, under the gluteal musculature of Wistar rat), a T-shaped prosthesis aligned well with the course of the sciatic nerve. A TGA (Therapeutic Goods Administration, Australia) approved biocompatible silicone tubing of $4.5 \mathrm{~m}$ in length (Outer diameter: $2.7 \mathrm{~mm}$ \& wall thickness: $0.75 \mathrm{~mm}$ ) was used to manufacture T-shaped prostheses. The silicone tubing was cut into 15 pieces of equal length. In order to make a single prosthesis, each of the tubing pieces was further cut into two unequal pieces of lengths $40 \mathrm{~mm}$ and 260 $\mathrm{mm}$ respectively. A hole (1.2 $\mathrm{mm}$ in diameter) was drilled in the middle of the $40 \mathrm{~mm}$ tubing piece to make the crossbar of the prosthesis (figure 3a), and one end of the longer piece $(260 \mathrm{~mm})$ was glued to crossbar in such a way that it covered the hole completely (figure $3 \mathrm{~b}$ ). To ensure there was no blockage at the junction, a metallic wire of $2 \mathrm{~mm}$ in diameter was passed through the crossbar and across the joint. Finally, the nerve prosthesis underwent a bubble detection test to ensure no leakage across the T-junction.

\subsection{Estimating tensile strength of sciatic nerve of a Wistar rat}

Sciatic nerves $(\mathrm{n}=12)$ were excised from fresh cadaveric Wistar rats (see figure 4a). Each nerve diameter was

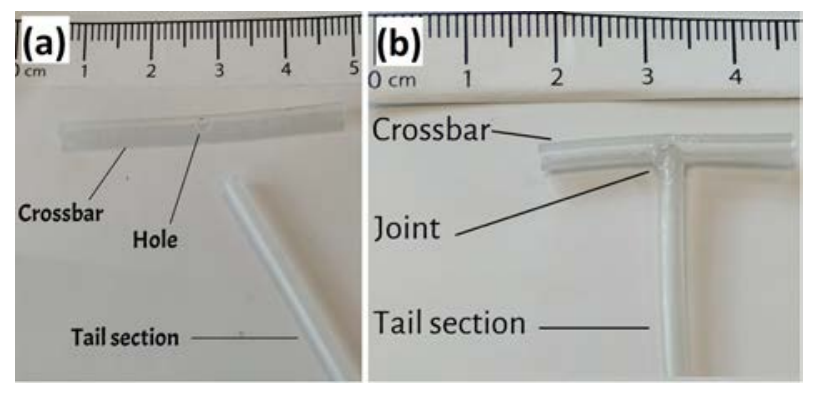

Figure 3. A T-shaped nerve prosthesis (The tail section is not fully shown.) 


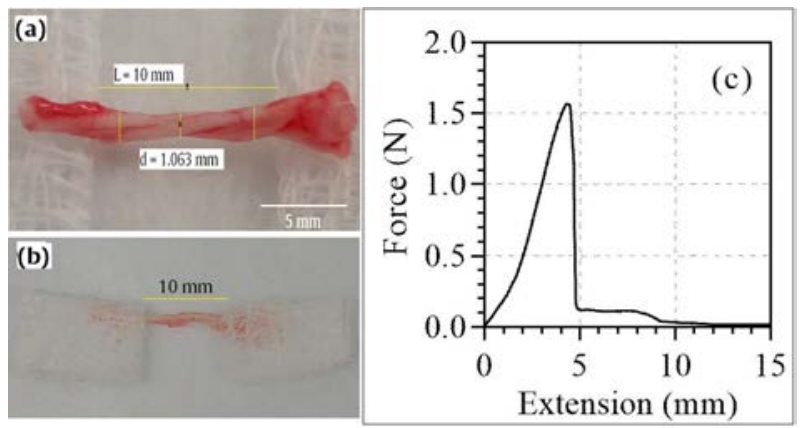

Figure 4. (a) An excised sciatic nerve. (b) The stumps of the sciatic nerve are wrapped in cotton gauze. (c) Force-extension graph of the sciatic nerve.

measured at three different locations along its length and averaged using an image processing software ImageJ_1.52a [13]. Both ends of the nerve were wrapped in a cotton gauze swab (figure $4 \mathrm{~b}$ ) to avoid non-uniform gripping and the sample was immobilized between two mechanical clamps in such a way that the final length between the two clamps was $10 \mathrm{~mm}$. Petroleum jelly was applied to the sample to avoid nerve desiccation. The tensile testing machine (TytronTM 250, MTS Systems Co., USA) was calibrated at $\pm 25 \mathrm{~N}$ and \pm $2.5 \mathrm{~N}$ according to the manufacturer's guidelines prior to testing. The nerve sample was elongated to failure at a strain rate of $10 \mathrm{~mm} / \mathrm{min}$, and a force-extension graph was obtained (figure 4c).

\section{Validating the performance of nerve stretcher}

Cadaveric Wistar rats were used to qualify the performance of the device. These experiments were carried out proactively to ensure the feasibility of the surgical procedure for implanting the T-shaped nerve prosthesis, finding an optimal method of keeping nerve stumps inside it during nerve stretching, and estimating the safe limit of applied vacuum to the nerve stumps. The left sciatic nerve was exposed in the midthigh region (figure 6c) and transacted using a scalpel. Proximal and distal nerve stumps were trimmed to ensure a gap of at least $10 \mathrm{~mm}$ between the two nerve ends.

The proximal and distal nerve stumps were inserted into the crossbar up to $5 \mathrm{~mm}$ from each end, and the tail of the prosthesis was connected via an externally mounted saddle to a pressure port (figure 5a). The vacuum was applied to the nerve stumps through this port, and the whole experimental setup (rat connected with a nerve stretcher) was placed in a biosafety cabinet. Starting at a pressure of $10 \pm 1 \mathrm{mmHg}$ - the minimum amount of vacuum the nerve stretcher can generate precisely - the device was programmed to increase the amount of vacuum $\left(\mathrm{P}_{\text {set }}\right)$ in $10 \mathrm{mmHg}$ intervals after every 5 hours; which in turn increased the stretching force applied to the nerve stumps. Figure 5b demonstrates the performance of the device in generating and maintaining a set vacuum over a fixed interval of time. These experiments also addressed the stability of nerve stumps inside the prosthesis. It was expected that the nerve stumps would move out of the prosthesis in live rats. Therefore, to overcome this limitation, a further two methods were devised (see section 5).

\section{Strategies to keep nerve stumps in the prosthesis}

A series of experiments were carried out in accordance with an approved animal ethics protocol (NRS/01/17/AEC). A total of 9 Wistar adult male rats, weighing 410 - 500 grams, postnatal age $\approx$ three months were used in this study. All surgical tools were autoclaved while the T-shaped nerve prosthesis was soaked in $100 \%$ ethanol for five minutes and then flushed with sterile saline $(0.9 \%$ Sodium Chloride, Pfizer Inc., USA). The rats were anaesthetised with a mixture of $\mathrm{O}_{2}$ /isoflurane (0.5L/1-3\%) at $2 \%$ induction and $1 \%$ maintenance along with subcutaneous buprenorphine $(0.05 \mathrm{mg} / \mathrm{kg} \mathrm{SC})$ injection to mitigate postoperative pain.

\subsection{Surgical Procedure}

The surgical sites were shaved, and antiseptic iodine (10\% Povidone-iodine solution) was applied to the shaved areas. The rat was positioned prone onto a sterilised drape over a heat blanket. The stabilisation of the hind limb was achieved using an adhesive tape. By using a skin marker (WriteSite 2900BN, Aspen Surgical Co., USA), three dots were made at the knee joint, hip joint, and ischial tuberosity respectively (figure 6a). A line $\left(\mathrm{L}_{1}\right)$ was drawn from the hip joint to ischial tuberosity. Similarly, another line $\left(\mathrm{L}_{2}\right)$ was drawn from the midpoint of $\mathrm{L}_{1}$ towards the knee joint, perpendicular to $L_{1}$, representing the course of the sciatic nerve. Finally, a skin incision was made along the dotted line $\left(\mathrm{L}_{3}\right)$ starting from the knee joint to the ischial tuberosity. The plane between biceps femoris and the gluteus maximus muscle was dissected bluntly using the muscle splitting approach (figure $6 \mathrm{~b}$ ) to reveal the underlying sciatic nerve. Retractors were placed beneath the muscles to widen the dissected area (figure 6c). 


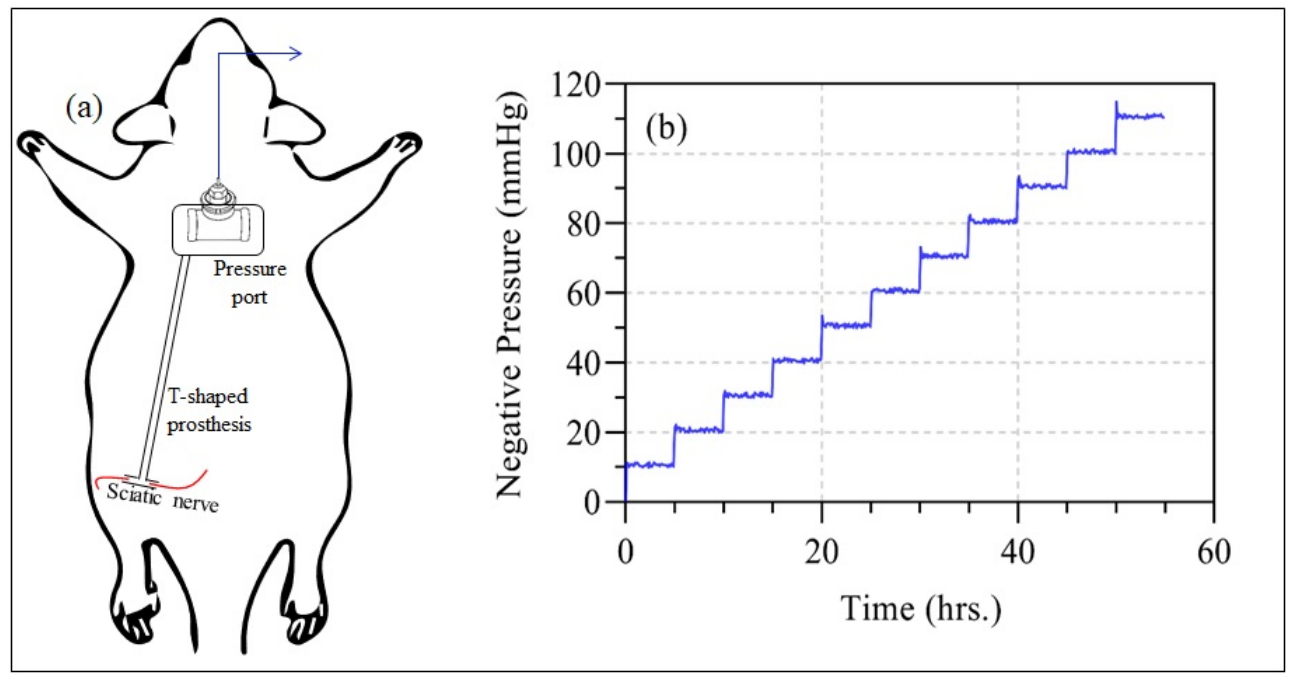

Figure 5. A line plot showing the performance of the device in maintaining vacuum.
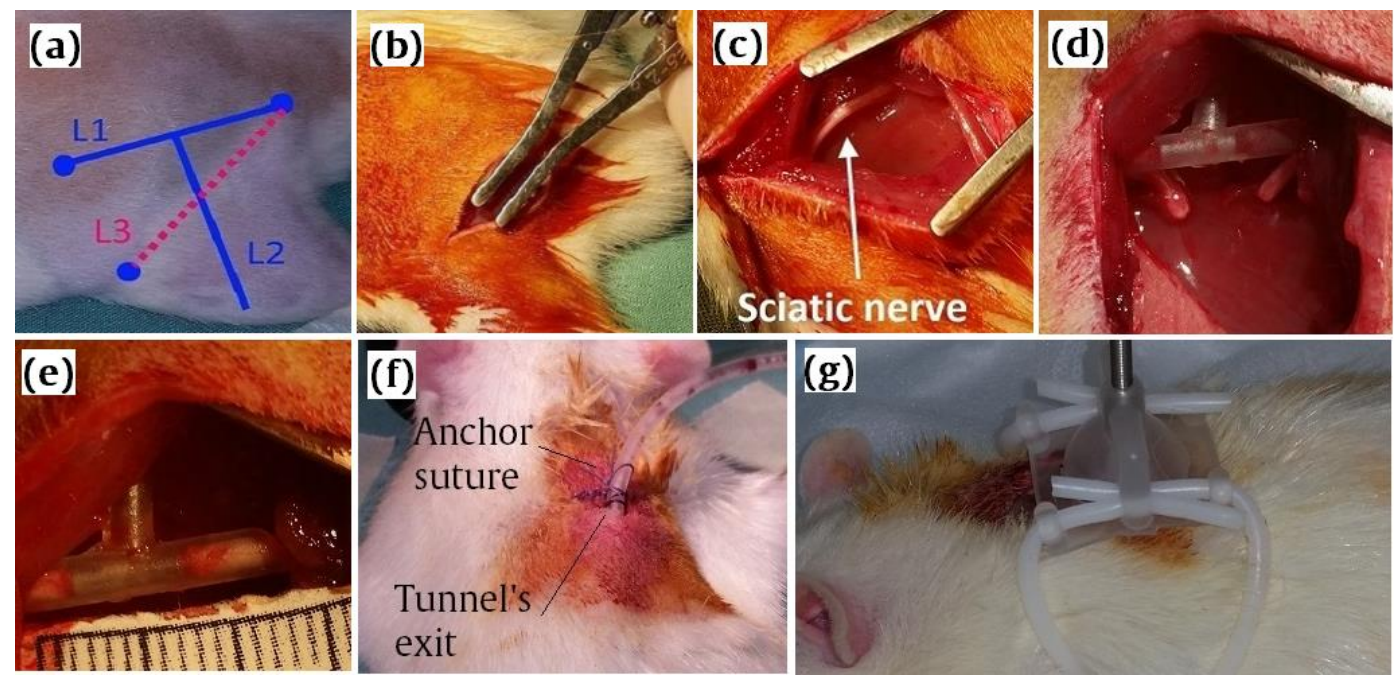

Figure 6. Surgical procedure and implantation of the prosthesis: (a) An operative technique for exposing the sciatic nerve of a Wistar rat. (b) A muscle splitting approach. (c) A retractor was used to widen the surgical area. (d) Crossbar aligned with the resected nerve. (e) Nerve ends are inside the crossbar of the prosthesis. $(f)$ An anchor suture at the tunnel's exit. $(g)$ Tunnel's exit is protected by a saddle.

\subsection{Sciatic nerve resection and implantation of the prosthesis}

The sciatic nerve was gently dissected from the surrounding connective tissues with curved scissors and resected using a microblade at its midpoint. The nerve ends were further excised to make a gap of $\approx 10 \mathrm{~mm}$ between them, aligned with the crossbar (figure $6 \mathrm{~d}$ ), and finally inserted into the respective ends of the nerve prosthesis (figure 6e). A subcutaneous tunnel was developed using a blunt rod starting from gluteal region, travelling subcutaneously and ending at the upperthoracic region. The tail of the prosthesis was towed away into the tunnel and secured to the skin at the tunnel's exit with an anchor suture (figure 6f) to prevent the dislocation of the prosthesis. The wound was irrigated with sterile saline and closed using absorbable sutures (6/0 PDS). A wound dressing spray (Opsite, Smith\&Nephew, AU) was also applied to the surgical areas to facilitate wound sterility, prevent maceration, and mitigate contamination.

The rat was tethered to a cage using a harness (Instech Lab Inc., USA) which consists of a softly moulded elastomeric saddle with a vented dome that protects the thoracic region from direct physical access, adjustable belly bands to secure the saddle to rat (figure $6 \mathrm{~g}$ ), and a spring stock to protect the externalised silicone tubing and transmit torque to a plastic swivel. One end of a silicone tubing was attached to the swivel while the other end, passing through the spring and 
exiting in the dome, was connected to the tail of the prosthesis coming out of the thoracic region using a straight tubing-connector (Part No. 11684, Qosina, Co. USA). The swivel was then mounted on a single-axis counter-balanced swivel mount (cm375bp, Instech Lab Inc., USA) and its other end was connected to the vacuum chamber.

\subsection{Applying vacuum to nerve stumps}

At this stage, the rats were divided randomly into three equal groups $(\mathrm{n}=3)$. All the rats received a continuous vacuum of $10 \mathrm{mmHg}$ for six days along with the following additional treatment to hold the nerve stumps inside prosthesis so that they did not slip out during rat locomotion.

- Group A: no additional support was provided (Control group).

- Group B: the nerve stumps were secured with an epineurial suture at each end of the crossbar.

- Group C: the same procedure as for group B along with the use of tissue adhesive to restrict the gliding of prosthesis over the soft tissues. Here, the tissue adhesive has no role other than to keep nerve prothesis in its place.

Rats were put in their respective cages, and vacuum $\left(\mathrm{P}_{2}\right)$ was applied to the nerve stumps and recorded every minute which resulted in 9640 values at the end of a single animal trial. Rats were provided with free access to food and water and exposed to 12 hours of light and dark cycles. At sixth day post-surgery, the rats were anaesthetised, and the surgical site was re-opened using the same procedure as described in section 5.1. The rats were sacrificed by subcutaneous lethabarb euthanasia injection (Virbac Pty Ltd, AU) concurrent to isoflurane.

\section{Results and Analysis}

\subsection{Biomechanical characterisation of sciatic nerves}

Figure $4 \mathrm{c}$ shows a force-extension graph of the sciatic nerve obtained as a result of tensile-test. The maximum stress that can damage the nerve was deemed to be $1.77 \mathrm{~N} / \mathrm{mm}^{2}$ which is close to $2.5 \mathrm{~N} / \mathrm{mm}^{2}$ [14] and 2.72 $\mathrm{N} / \mathrm{mm}^{2}[15]$ reported in the literature. In the present research, $1.77 \mathrm{~N} / \mathrm{mm}^{2} \pm 0.3 \mathrm{~N} / \mathrm{mm}^{2}$ is maximum stress a sciatic nerve can withstand; values exceeding this could irreversibly damage the nerve physically and were avoided (discussed in section 6.2). However, there is no risk involved in crossing this threshold as the nerve stretcher, even if run continuously, couldn't generate vacuum higher than $0.0267 \mathrm{~N} / \mathrm{mm}^{2}$.

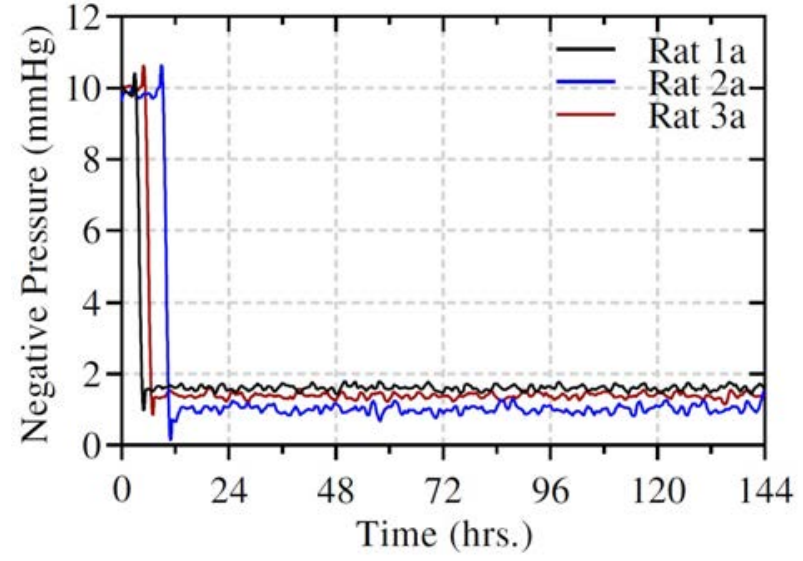

Figure 7. Vacuum history of rats in group-A.

\subsection{Analysis of pressure data from cadaveric rats}

Figure $5 \mathrm{~b}$ demonstrates the performance of the device in maintaining a vacuum in the vacuum chamber. The level of vacuum was set to increase by $10 \mathrm{mmHg}$ every 5 hours until it reached to $110 \mathrm{mmHg}\left(0.01467 \mathrm{~N} / \mathrm{mm}^{2}\right)$. At this pressure, the axoplasmic fluid was observed in the crossbar of the prosthesis. The device was stopped immediately, and by fine-tuning the vacuum, the threshold $\left(\mathrm{P}_{\text {safe }}\right.$ ) was found to be $102 \mathrm{mmHg}$ $\left(0.0136 \mathrm{~N} / \mathrm{mm}^{2}\right)$ illustrating that in order to avoid the loss of intra-neural fluid and subsequently collapsing the nerve's internal structural framework, the set pressure $\left(\mathrm{P}_{\text {set }}\right)$ must not exceed limiting pressure $-\mathrm{P}_{\text {safe }}$. Thus, the lower and upper limit of vacuum was set to 10 $\mathrm{mmHg}$ and $100 \mathrm{mmHg}$ respectively which corresponded to forces of $1.2 \mathrm{mN}$ and $11.8 \mathrm{mN}$ acting on the nerve stumps in the prosthesis.

\subsection{Analysis of pressure data of live rats}

Figure 7 shows the negative pressure for the three rats in group $\mathbf{A}$ whose nerve ends were pulled in the crossbar without additional securing. Once the animals awoke from surgery, the nerve stumps dislocated from the prosthesis - as expected -, and a significant leak in pressure was observed. It is apparent that for the first and third rats, the pressure dropped immediately while for the second rat, it dropped around the $12^{\text {th }}$ hours post-surgery. On examining the surgical site, the proximal, as well as distal nerve stumps of the first and third rats, were found out of prosthesis. In contrast, only the proximal nerve stump of the second rat was seen out of prosthesis. Due to the dislocation of the nerve stumps, wound exudate was also sucked from the injury site and observed in the prosthesis's tail-section.

The nerve stumps of the sciatic nerves of rats in group $\mathbf{B}$ were secured to their prosthesis by suturing epineurium (8/0 Nylon) to the ends of the crossbar 

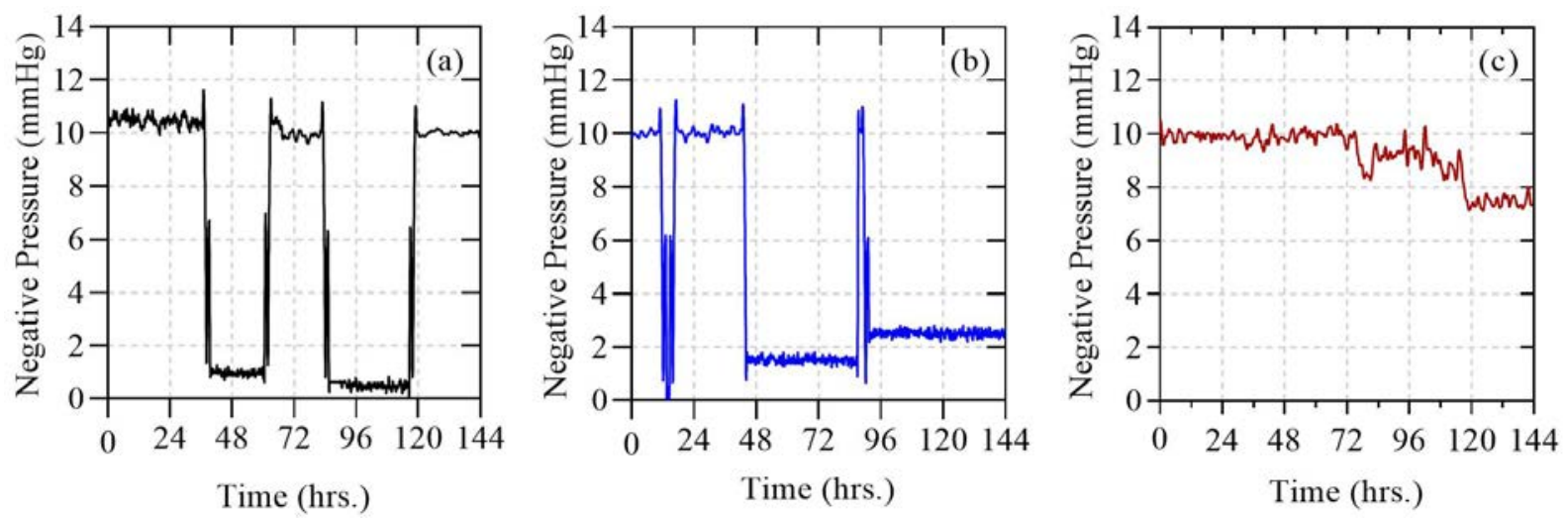

Figure 8. Vacuum history of rats in group B. $(a \& b)$ - Frequent pressure dropped. (c) Pressure remained stable but fluctuated significantly.

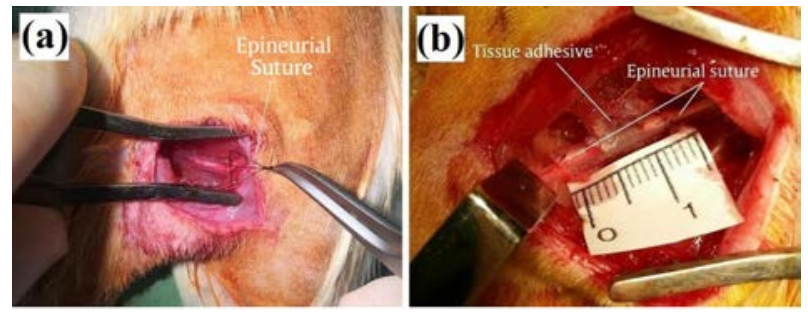

Figure 9. (a) Group B rats: Epineurial suture at ends of the crossbar. (b) Group-C rats: Epineurial sutures along with the use of tissue adhesive.

of each prosthesis (figure 9a). In figure 8, the graphs show a noticeable fluctuation in pressure for all rats in this group. For instance, a significant drop in vacuum was observed for rats $1 b$ and $2 b$ (figure $8 \mathrm{a}$ and figure $8 \mathrm{~b}$ ) throughout the duration of the experiment. It seemed that the inability of the prosthesis to hold the nerve stumps in their place is a contributing factor to this unpredictable fluctuation. On postmortem examination, these three rats, the distal nerve stumps remained secure in the prosthesis while proximal nerve stumps were found outside the prosthesis but still attached to the epineurial suture. For rat $3 \mathrm{~b}$ (figure 8c), a stable pressure was recorded, and both nerve stumps were found inside the prosthesis on postmortem. Interestingly, this seemed to correlate to the activity of the animal, as rat $3 \mathrm{~b}$ displayed the least activity for the first three days as compared to the others, without signs of fear, pain or abnormal posturing. However, vacuum data shows a considerable deviation from mean value suggesting that although epineurial sutures may secure the nerve stumps to some extent they eventually fail after longer durations.

The rats in group $\mathbf{C}$ received the same suturing as did the rats in group $\mathbf{B}$ along with additional tissue adhesive. The tissue adhesive (Histoacryl, B. Braun AG, DE), consisting of monomeric n-butyl-2cyanoacrylate as an active ingredient, was used to adhere the junction of the prosthesis to adjacent tissues in order to restrict the excursion of the prosthesis during locomotion (figure 9b). The additional support that the tissue adhesive provided to the prosthesis resulted in a stable axial stretch for all the rats in this group (figure 10). The axial force was measured to be $10 \pm 0.05$, $9.9 \pm 0.06$, and $9.9 \pm 0.08 \mathrm{mmHg}$ for rat $1 \mathrm{c}, 2 \mathrm{c}$ and $3 \mathrm{c}$ respectively. The proximal and distal nerve stumps of all the rats were found entirely within the prosthesis at postmortem examination. Macroscopically, there were no signs of tissue inflammation due to the use of tissue adhesive. In general, the rats were found healthy and displayed normal signs as specified in the ethics protocol. Since the pressure remained constant for all the rats in this group, the distribution of pressure data was presented in the form of a box-and-whisker. The vacuum data for each rat is divided into four quartiles Q1, Q2, Q3 and Q4. A shorter distance, as in Q4 of rat 1c, illustrates that there were negligible fluctuations in the vacuum whereas the opposite is true for a longer distance, as in Q4 of rat 2c. The centre line of each box shows the average value of vacuum $\left(\mathrm{P}_{2}\right)$ which is reasonably close to the desired value $\left(\mathrm{P}_{\text {set }}\right)$. This suggests that there was no pressure leakage or slipping of nerve stumps out of prosthesis.

\section{Conclusion}

In summary, a method of applying an in vivo axial tensile force to nerve fibres within a whole peripheral nerve (sciatic nerve) using a controlled vacuum has been presented. A device named nerve stretcher was designed and constructed to apply vacuum to nerve stumps in a custom-made T-shaped nerve prosthesis. Preliminary experimentation on cadaveric Wistar rats successfully demonstrated the performance of the nerve stretcher in generating and providing a stable vacuum. Further experiments on live rats revealed the potential for different strategies to hold the nerve 


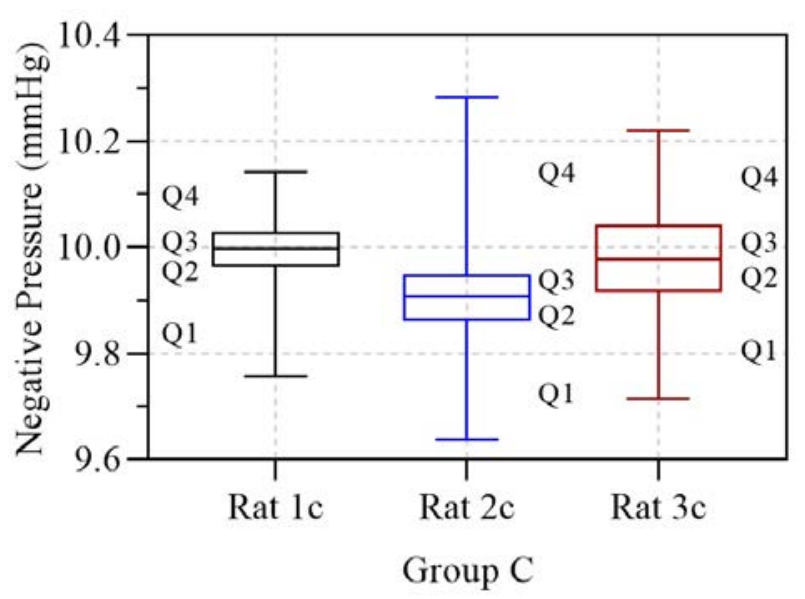

Figure 10. Vacuum history of rats in group-C.

stumps in the prosthesis. The experimental results infer that the strategies used in group $\mathbf{A}$ and $\mathbf{B}$ are not capable of maintaining the nerve stumps in place and they subsequently became displaced once rats resumed locomotion. However, as in group $\mathbf{C}$, the stability of the nerve stumps was achieved successfully by restricting the degree of freedom of the prosthesis using tissue adhesive; the use of tissue glue facilitated the nerve stumps to remain in the prosthesis which in turn proved to be effective in providing a stable vacuum to the sectioned nerve stumps.

The observations obtained from the experimental results proposed an effective technique to hold nerve stumps in a prosthesis, demonstrate the effectiveness of the developed platform, and provide guidelines for the future exploration of nerve stretch-growth studies. Moreover, the nerve stretcher proved to be a viable option for applying controlled axial stress to a peripheral nerve in vivo. In future studies, on the basis of the current findings, the device will be tested in respect to its ability to enhance peripheral nerve regeneration.

\section{Acknowledgments}

The authors did not receive any financial support for this work that could have influenced its outcome. The authors also declare no conflicts of interest related to this manuscript.

\section{References}

[1] Weiss P and Hiscoe H B 1948 Journal of Experimental Zoology 107 315-395 ISSN 0022-104X URL http://doi. wiley.com/10.1002/jez.1401070302

[2] Smith D H 2009 Progress in Neurobiology 89 231-239 ISSN 0301-0082 URL https://www.sciencedirect. com/science/article/pii/S0301008209001099? via\{\%\}3ihub

[3] Weiss P 1941 Nerve patterns : the mechanics of nerve growth Third Growth Symposium pp
163-203 URL https://www.worldcat.org/title/ nerve-patterns-the-mechanics-of-nerve-growth/ oclc/537059321

[4] Bray D 1984 Developmental Biology 102 379-389 ISSN 00121606 URL https://www.sciencedirect.com/science/ article/pii/0012160684902021?via\{\%\}3ihub

[5] Lamoureux P, Buxbaum R E and Heidemann S R 1989 Nature 340 159-162 ISSN 0028-0836 URL http://www. nature.com/articles/340159a0

[6] Zheng J, Lamoureux P, Santiago V, Dennerll T, Buxbaum $\mathrm{R}$ E and Heidemann S R 1991 The Journal of neuroscience : the official journal of the Society for Neuroscience 11 1117-25 ISSN 0270-6474 URL http: //www.ncbi.nlm.nih.gov/pubmed/2010807

[7] Lamoureux P, Heidemann S R, Martzke N R and Miller K E 2010 Developmental Neurobiology 70 135-149 ISSN 19328451 URL http://doi.wiley.com/10.1002/ dneu. 20764

[8] Dennerll T J, Joshi H C, Steel V L, Buxbaum $\mathrm{R}$ E and Heidemann S R 1988 The Journal of cell biology 107 665-74 ISSN 0021-9525 URL http://www.ncbi.nlm.nih.gov/pubmed/3417767http: //ww. pubmedcentral.nih.gov/articlerender.fcgi? artid=PMC2115196

[9] Smith D H, Wolf J A and Meaney D F 2001 Tissue Engineering $\mathbf{7}$ 131-139 ISSN 1076-3279 URL http://www.ncbi.nlm.nih.gov/pubmed/11304449http: //www.liebertonline.com/doi/abs/10.1089/ 107632701300062714

[10] Pfister B J, Iwata A, Meaney D F and Smith D H 2004 Journal of Neuroscience 24 7978-7983 ISSN 0270-6474 URL http://www. jneurosci.org/cgi/doi/10. 1523/JNEUROSCI . 1974-04.2004

[11] Pfister B J, Iwata A, Taylor A G, Wolf J A, Meaney D F and Smith D H 2006 Journal of Neuroscience Methods 153 95-103 ISSN 01650270 URL https://www.sciencedirect.com/science/ article/pii/S0165027005003663?via\{\%\}3Dihub

[12] Pfister B J, Bonislawski D P, Smith D H and Cohen A S 2006 FEBS Letters 580 3525-31 ISSN 00145793 URL http://www.ncbi.nlm.nih.gov/pubmed/16730003http: //www.pubmedcentral.nih.gov/articlerender.fcgi? artid=PMC5367051http://doi . wiley. com/10.1016/j . febslet.2006.05.030

[13] Schneider C A, Rasband W S and Eliceiri K W 2012 Nature Methods 9 671-675 ISSN 1548-7091 URL http: //www.nature.com/articles/nmeth.2089

[14] Abrams R A, Butler J M, Bodine-Fowler S and Botte M J 1998 The Journal of Hand Surgery 23 465-470 ISSN 03635023 URL https://www.sciencedirect.com/science/ article/pii/S0363502305804642?via\{\%\}3Dihub

[15] Borschel G H, Kia K F, Kuzon W M and Dennis R G 2003 Journal of Surgical Research 114 133-139 ISSN 0022-4804 URL https://www.sciencedirect.com/ science/article/pii/S0022480403002555 